\title{
Stress Interactions at the Cellular and Membrane Levels
}

\author{
Jiwan P. Palta \\ Department of Horticulture, University of Wisconsin, Madison, WI 53706
}

In nature, plants are often exposed to several environmental stresses at any given time. For example, chilling or heat stress is often accompanied by water stress and, during winter, evergreens are often exposed to light (photo-oxidation) stress. Thus, to better-understand plant response to the environment under natural conditions, one must learn the mechanisms by which the plant adapts to, is injured by, and recovers from various environmental stresses. One approach to understand these interactions is to study the nature of stresses and stress-induced perturbations at the cellular and membrane levels. Membranes have been implicated as sites of response by various environmental stresses for a long time (see Levitt, 1980). However, only recently has the nature of these responses at the membrane level been studied. In this paper, I have attempted to synthesize the research, especially in the area of freezing stress. Research on membrane aspects is vast and beyond the scope of this paper. I have tried to focus on some key aspects of cell membranes (especially plasma membrane) important in the initiation and progression of injury. The present status of research is discussed to point out future directions of research that might bring better understanding of the interactions among various environmental stresses.

\section{Process of dehydration: Loss of water}

A common feature among environmental stresses is dehydration. Extracellular water in land plants contains a much lower concentration of solutes than the vacuolar (cell sap) water. This concentration difference, combined with the semipermeable nature of the cell membranes and the relatively rigid cell wall, is responsible for generating turgor pressure. As the tissue dehydrates beyond complete loss of turgor (wilting), the process of dehydration is similar in freezing stress and water stress, which are different from osmotic stress (Fig. 1).

Freezing stress. Air cooling rates during a frost episode are generally 1 to $2 \mathrm{C} / \mathrm{hr}$. Extensive examination of climatological data for various locations in Wisconsin revealed that air cooling rates in the subzero range generally do not exceed 2C/hr (Steffen et al., 1989). Ice initiation in the tissue requires nucleation, and the plant tissue is usually nucleated by the time tissue temperatures drop to -1 to - 3C (Ashworth et al., 1985; Steffen et al., 1989). Slow cooling ( 1 to $2 \mathrm{C} / \mathrm{hr}$ or slower) results in ice initiation in the extracellular water, because extracellular water has a lower solute concentration than intracellular water, and because ice nucleators such as dust, bacteria, etc. are prevalent in the extracellular environment. As air temperature drops further, ice grows in the extracellular space, withdrawing water from the cell and resulting in collapse of the cell walls (Fig. 1). Recent direct microscopic examination of the frozen tissue supports this conclusion (Pearce, 1988). Water moves from the inside to the outside of the cell because the vapor pressure of the extracellular ice is lower than the vacuolar and cytoplasmic water at the same temperature. Thus, the plasma membrane does not separate from the cell wall during extracellular freezing stress (Fig. 1) (Pearce, 1988).

Water stress. The process of dehydration by water stress is similar to extracellular freezing stress, except that the extracellular space contains air in water-stressed tissue rather than ice as in the freezestressed tissue. As the plant tissue loses water, the water is first removed from the larger pores and capillaries in the cell walls. At each stress level, the water potential of the vacuole and cytoplasm remains in equilibrium with the water potential of the pores in the cell wall. Thus, similar to freezing stress, the plasma membrane does not separate from the cell wall during water stress (Fig. 1).

\footnotetext{
This manuscript was based on research supported by a USDA competitive grant (Agreement no. 85-CRCR-1-1673) and by the College of Agriculture and Life Sciences, Univ. of Wisconsin, Madison.
}

This observation was made by many early workers using light microscopy (see Levitt, 1980).

Osmotic stress. As the concentration of the extracellular solution becomes hypertonic, the plasma membrane separates from the cell wall (Fig. 1). This separation is called plasmolysis, and takes place in a hypertonic solution of a non- (or slowly) permeating solute. Shrinkage of protoplasm (protoplasm and vacuole) with the removal of water has been demonstrated to follow the Boyle-Mariotte-van't Hoff law (Palta and Lee-Stadelmann, 1983). The space between the plasma membrane and the cell wall is occupied by the extracellular solution. Thus, a reduction in the surface area of the plasma membrane occurs upon plasmolysis, which does not-occur in cells exposed to freezing and water stress.

Interrelationships among stresses. Stresses imposed on a plant cell by freezing, water, or osmotic stress are different. The common stress among these three is loss of water from the cell. In freezing stress, collapse of the cell wall results in a mechanical stress on the cell in addition to low temperature and an increase in the concentration of organic and inorganic solutes. Stresses imposed on cells undergoing water stress are similar to freezing stress, except that they occur at higher temperatures in the former. In support of these ideas, onion epidermal cells were found to survive temperatures as low as $-20 \mathrm{C}$ and equivalent desiccation stress (Palta et al., 1977c). There is no mechanical stress imposed on cells exposed to osmotic stress. This point is important because some studies have claimed that freezing stress can be simulated by subjecting isolated protoplasts to osmotic stress (Steponkus and Weist, 1978). Conclusions drawn from such studies regarding the perturbation of cell membranes by freezing stress cannot be valid because membranes are exposed to very different kinds of stresses with osmotic stress. In several other studies, protoplasts have been used as a model system to investigate the mechanism of freezing injury (Steponkus et al., 1982; Dowgert and Steponkus, 1984; Steponkus, 1984). Since protoplasts are bathed in an isotonic external solution, the freezing stress is essentially an osmotic stress in this system. Protoplasts provide opportunities for experimental manipulation that are not possible with intact cells. However, use of protoplasts eliminates totally the role of the cell wall. In support of this criticism, a large difference in the freezing tolerance of intact cells and their protoplasts was recently found (Tao et al., 1983). Released protoplasts had $\mathrm{LT}_{50}$ of $-21.5 \mathrm{C}$, as compared to $-12 \mathrm{C}$ for intact cells suspended in the same medium. These results highlight the importance

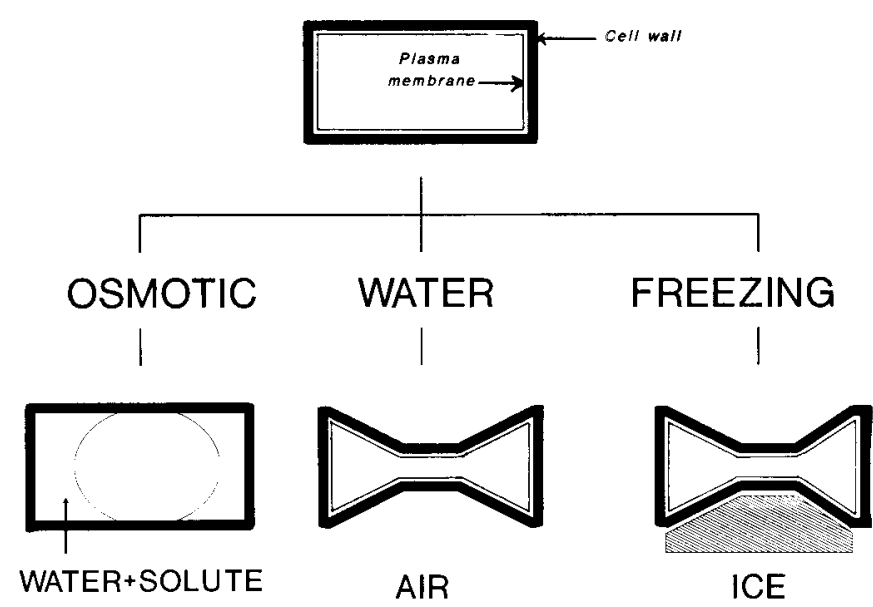

Fig. 1. A diagrammatic description of the process of dehydration at the cellular level by freezing, water, and osmotic stresses. 
of using intact tissues and cells for the study of stress-induced injury.

\section{Mechanism of injury}

The visual symptoms of stress-induced injury are often a watersoaked appearance, leakage of solutes, and the inability to regain turgor upon removal of the stress. These symptoms suggest the cell membrane is a site of damage. Changes in the cell membrane properties following a freeze-thaw stress have been studied extensively. Until recently, it was thought that freezing stress results in a total loss of membrane semipermeability and in membrane rupture. However, systematic examination of freeze-thaw-stressed water-soaked tissue has revealed that solute leakage occurs from injured, yet alive, cells. In spite of a large increase in ion and sugar leakage, the cells were able to plasmolyze, exhibit protoplasmic streaming, and could be vitally stained with fluorescein diacetate (Arora and Palta, 1988; Palta et al., 1977a, 1977b). Furthermore, various organelle functions have differential sensitivity to freezing stress. For example, using potato leaf tissue, we have found photosynthesis to be much more sensitive to freezing stress than respiration (Fig. 2) (Steffen et al., 1989). In these studies, the first cellular perturbation was found to be a 2.5-fold increase in ion leakage, followed by a drop in photosynthesis (Fig. 2). Increased ion leakage suggests perturbation of the transport properties of the cell membranes that would result in an altered cellular environment. In spite of this altered environment, no changes in the ultrastructure of the chloroplasts and mitochondria were observed at incipient injury, while swelling and ultrastructural disorganization were observed following a lethal freeze-thaw stress (Palta et al., 1982; Palta and Li, 1978a; 1978b). These studies have established that early events in freeze-thaw injury are subtle rather than cataclysmic.

Membrane pumps. as a site. of injury. As discussed above, membrane perturbation is an early manifestation of freezing injury. We have proposed that a sublethal freeze-thaw stress results in alterations of the transport properties of the cell membranes (Palta and $\mathrm{Li}, 1978 \mathrm{a}, 1980)$. These alterations result from functional and/or structural changes in the membrane associated $\mathrm{H}^{+}$-ATPase (membrane pumps). This hypothesis is based on several investigations of freeze-injured, yet living, cells (Palta et al. 1977a, 1977b; Palta and Li, 1980; Palta et al., 1982). The results of these studies show that following sublethal freeze-thaw injury: a) The major cation that leaks out of the cell is $\mathrm{K}^{+}$, and the transport of $\mathrm{K}^{+}$is known to be coupled to $\mathrm{H}^{+}$-ATPases (Sze, 1985); b) there is a small, but significant, loss of cellular $\left.\mathrm{Ca}^{++} ; c\right)$ there is no change in the membrane permeability to water and nonelectrolytes, suggesting intactness of membrane lipids; and d) depending on the degree of initial injury, there is a possibility of complete repair or recovery. Recov-

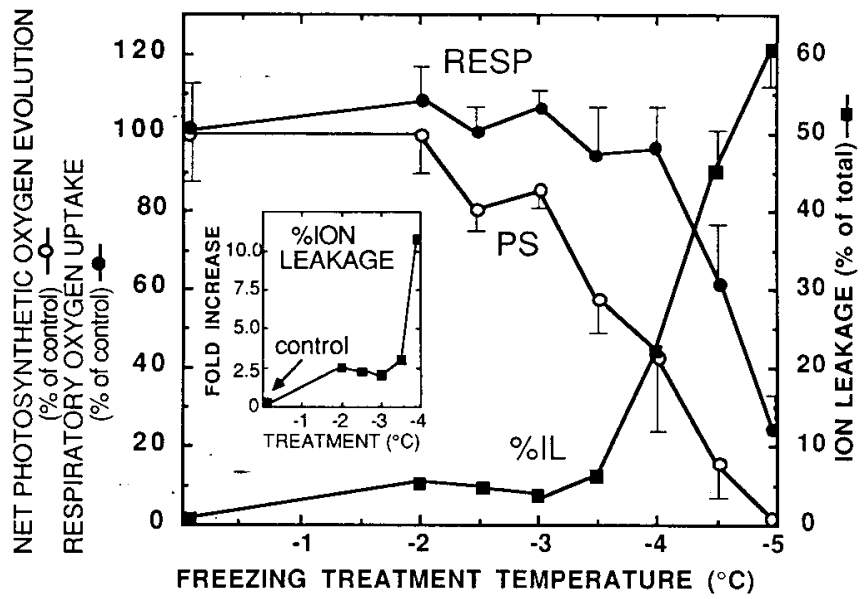

Fig. 2. Differential sensitivity of photosynthesis, respiration, and cellular membranes [measured as percentage of ion leakage (IL)] to extracellular freezing stress in leaf tissue of Solanum commersonii L. Excised leaflets were frozen to desired temperature at a cooling rate of $1 \mathrm{C} / \mathrm{hr}$ with ice nucleation at $-0.5 \mathrm{C}$. Following slow thaw, various cellular functions were measured on the same leaflet (Steffen et al., 1989.) ery of injury is one of the important aspects of these results. It was found that, depending on the degree of injury, symptoms of water soaking were completely reversible. This means that cells have the capability to repair the damage. Since recovery requires the uptake of all leaked solutes, activity of the membrane ATPase is needed for this to occur.

We recently have found that the specific activity of ATPase associated with the plasma membrane is increased following reversible injury (Iswari and Palta, 1989). This supports our reasoning that activity of plasma membrane ATPase is needed for recovery. In this study, we also found that activity of ATPase decreases with increasing injury, whereas the activity of several other key enzymes such as cytochrome C-oxidase and cytochrome c-reductase were found to be stable following lethal injury. These results suggest that plasma membrane ATPase is an early and more-sensitive site of alteration following freeze-thaw injury. How the functions of membrane proteins such as ATPase are altered is currently being investigated. Some possibilities include direct protein denaturation, confirmational alteration of membrane proteins, changes in the lipids closely associated with this protein (lipid-protein interaction), changes in the membrane and/or cytosolic calcium, or changes in the membrane protentials (Iswari and Palta, 1989).

Role of cellular/membrane calcium. In recent years, it has become clear, after the discovery of calmodulin, that $\mathrm{Ca}^{2+}$ is not simply a macronutrient but a major metabolic and developmental controller (see review by Poovaiah and Reddy, 1987). Calmodulin is a calcium-dependent regulator protein found in both plants and animals. Together with calmodulin, or by itself, calcium can function as a secondary messenger in plant cells (Hepler and Wayne, 1985; Marine, 1982, 1983; Poovaiah, 1985; Poovaiah and Reddy, 1987). Stimuli, such as hormones, light, gravity, or other environmental conditions, can cause a slight increase in the calcium concentration of the cytoplasm (Fig. 3). When the cytoplasmic calcium level rises, calcium ions bind to calmodulin, thus activating the calmodulin. Only activated calmodulin is able to recognize the receptor protein (enzyme) and bind to it. This binding leads to the active calmodulin-calcium-enzyme complex that is capable of inducing cellular response. Since the calcium concentration in the cytoplasm is very low, small changes in the absolute amount of calcium can create 10- to 100-fold differences in concentration, without upsetting the ionic balance of the cell. This feature makes calcium an excellent secondary messenger. The combination of a relatively large ionic radius and low free energy of hydration (binds water rather weakly) contribute in part to the high degree of selectivity of calcium compared to other cations for its role as a secondary messenger (Poovaiah and Reddy, 1987). These recent findings provide exciting opportunities to study and understand interaction among environmental stresses.

SIGNAL

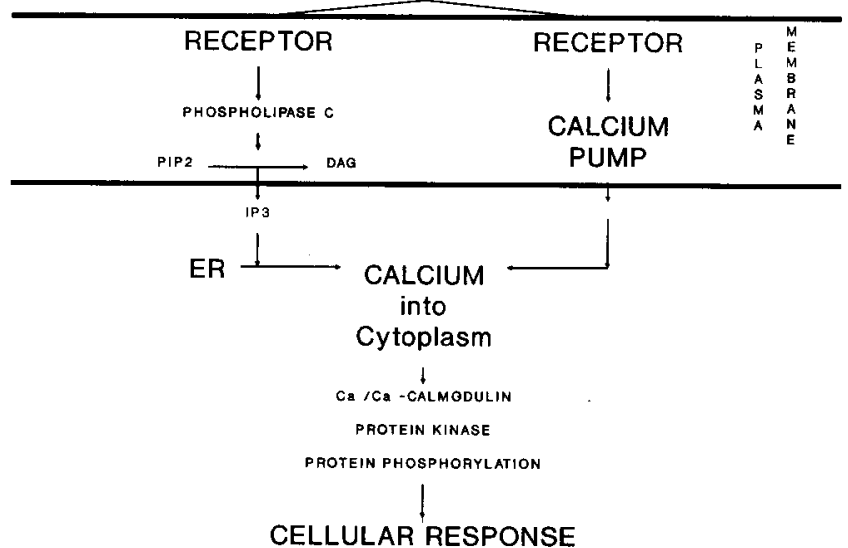

Fig. 3. A flow diagram illustrating the pathway by which cellular calcium changes can occur, which, in turn, result in cellular response to stress. Based on the scheme given by Poovaiah and Reddy (1987). PIP ${ }_{2}$ : phosphatidyl inositol4,5bisphosphate; DAG: diacylglycerol; $\mathrm{IP}_{3}$ : inositoll,4,5trisphosphate. 
We have reported evidence for the loss of membrane-associated calcium in the early stages of freezing injury (Arora and Palta, 1986, 1988). The major cation that leaks out of cells is $\mathrm{K}^{+}$, along with a small, but significant, amount of $\mathrm{Ca}^{2+}$ (Palta et al., 1977a). During the post-thaw period, irreversibly injured cells undergo protoplasmic swelling (Palta and Li, 1978a, 1980). This aberration is thought to be caused by perturbation of the cell membrane by high extracellular $\mathrm{K}^{+}$that accumulates following injury. We hypothesized that this perturbation results from the weakening of the cell membrane due to removal of membrane calcium by extracellular $\mathrm{K}^{+}$(Palta and Li, 1978a).

Using the outer epidermal layer of unfrozen onion (Allium cepa L.) bulb scales (anthocyanin-containing cells), we were able to simulate the irreversible freezing injury symptoms by bathing epidermal cells in $50 \mathrm{~mm} \mathrm{KCl}$ (Arora and Palta, 1986). Furthermore, extracellular $50 \mathrm{~mm} \mathrm{KCl}$ was shown to be injurious to the cells in this study. Addition of 10 to $20 \mathrm{~mm} \mathrm{CaCl}_{2}$ to the extracellular $\mathrm{KCl}$ solution prevented both the irreversible injury symptoms and the injurious effects of $\mathrm{KCl}$. In support of this finding, freeze-injured cells recovered much better when washed with $20 \mathrm{mM} \mathrm{CaCl}_{2}$ compared to unwashed controls (Palta et al., 1977a).

To test the hypothesis of perturbation of membrane-associated calcium due to freeze-thaw stress and extracellular $\mathrm{K}^{+}$, we used CTC (chlorotetracycline), a calcium-binding antibiotic, as a fluorescent probe for membrane $\mathrm{Ca}^{2+}$ (Arora and Palta, 1988). The fluorescent signal was quantified by using image analysis. Onion bulb scale cells were subjected to either reversible (incipient) or irreversible (lethal) freezing stress. A linear inverse relationship was found between freezing injury (ion leakage) and membrane-associated calcium (Fig. 4). Irreversible freezing injury caused a substantial loss of membrane-associated calcium. Even the reversibly injured cells showed loss of membrane-associated $\mathrm{Ca} 2+$. This loss of $\mathrm{Ca}^{2+}$ could be simulated by bathing non-frozen cells in $50 \mathrm{~mm}$ $\mathrm{KCl}$. Furthermore, this loss was prevented by having a small amount of $\mathrm{CaCl}_{2}$ in the bathing medium. Our results suggest that loss of membrane-associated $\mathrm{Ca}^{2+}$ plays an important role in the initiation and progression of freezing injury. Similar results have been reported for salt stress. In these studies, the protective role of $\mathrm{Ca}^{2+}$ was demonstrated against $\mathrm{NaCl}$ stress (Cramer et al., 1985) and a reduction in membrane associated calcium was found by salt $/ \mathrm{NaCl}$ stress (Lynch et al. 1987).

The scheme outlined in Fig. 3 describes how cellular response to environmental stress can be brought about by the perturbation of cytoplasmic calcium. It is tempting to explain recovery following stress by the perturbation of cytoplasmic $\mathrm{Ca}^{2+}$. As mentioned above, recovery requires activity of $\mathrm{H}^{+}$-ATPase to pump back the leaked ions. We have found activation of the plasma membrane associated

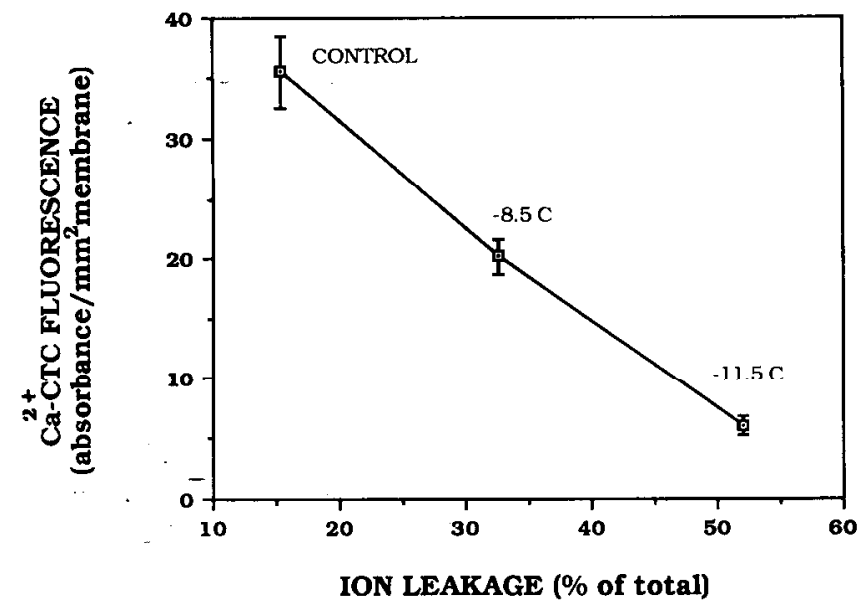

Fig. 4. Relationship between membrane damage (expressed as percentage of ion leakage) and membrane-associated calcium (measured as $\mathrm{Ca}^{2+}$. CTC fluorescence signal). Onion bulb scale tissue was subjected to slow freeze-thaw stress. Following thawing, the inner epidermal cells were stained with CTC (chlorotetracycline) fluorescent dye. The signal from this stain was quantified by image analysis (Arora and Palta, 1988.)
ATPase following incipient (reversible) injury (Iswari and Palta, 1989). Recently, it has also been demonstrated that a $\mathrm{Ca}^{2+}$-requiring protein kinase is able to modulate the plasma membrane-associated ATPase (Schaller and Sussman, 1988). Thus, it is possible that recovery, in part, is brought about by changes in cytoplasmic $\mathrm{Ca}^{2+}$ by activating a protein kinase (Fig. 4), which, in turn, activates plasma membrane ATPase. It appears that mediation of stress response via changes in cellular/membrane calcium will be an active and fruitful area of research in the near future.

Membrane lipids as receptor of the stress signal. The fluid-mosaic model regards the biological membrane as a two-dimensional solution of oriented proteins in a fluid bilayer phase (Singer and Nicholson, 1972). It is now apparent that membrane lipid has a very important role in determining the functions of membrane enzymes (for review, see Carruthers and Melchior; 1986). Features of the membrane bilayer that can alter the activity of transport proteins include lipid head groups, lipid acyl chain length, saturation/unsaturation, lipid backbone, and lipid fluidity.

A change in the physical state of the membrane lipids from a flexible liquid-crystalline structure to a solid gel-structure was proposed as a primary response to chilling injury (Lyons et al., 1964; Lyons and Asmundson, 1965; Lyons and Raison, 1970). This hypothesis has been highly controversial and has been extended to explain injury by freezing stress. Evidence for and against this hypothesis has been presented by. many researchers (for review, see Kuiper, 1985). Two approaches have been used to test this hypothesis. In one approach, the researchers attempted to relate the difference in lipid composition, lipid phase transition temperatures, lipid phase separation temperatures, and/or lipid "fluidity" (viscosity) to the chilling- or freezing-stress resistance of various unrelated plant species. In the other approach, changes in these lipid properties were investigated during low temperature acclimation. Part of the contradiction in these studies can be explained by the fact that some researchers used microsomal fractions or isolated organelles, whereas others used intact plant tissue for the study of lipid properties.

We have found that meaningful changes in lipid properties can only be studied by using purified membrane preparations of interest (Palta and Meade, 1987). Another criticism of many studies is that comparisons were made between genetically unrelated plants. We have found that a more-relevant relationship between lipid properties and stress resistance can be made by comparing genetically related plant species that vary in stress resistance (Palta and Meade, 1987).

Changes in calcium and dehydration can also bring about changes in the physical properties (fluidity) of the membrane lipids (Legge et al., 1982; Paliyath et al., 1984). In general, dehydration and increasing calcium concentration tend to rigidify the membrane lipid. A scheme summarizing the role of lipids in stress response is given in Fig. 5. As discussed above, there is sufficient evidence in the literature suggesting changes in membrane lipids from fluid to gellike structure as the temperature drops, as the calcium level of the membrane is perturbed, or as dehydration occurs. Such changes in lipid properties can result in denaturation or alteration in functions of the membrane-associated enzymes (Carruthers and Melchior, 1986). These changes, in turn, lead to cellular response (Fig. 5).

We have hypothesized that plasma membrane ATPase (pump) is a key site of stress response. We have found an increase in the activity of plasma membrane ATPase during cold acclimation (Iswari and Palta, 1989). In parallel with this increase in activity of ATPase, an increase in phospholipid fatty acid 18:2 (linoleic acid) and a decrease in 16:0 was found (Palta and Meade, 1987). There was, in general, also an increase in the unsaturation level of fatty acids, suggesting an increase in membrane "fluidity" after acclimation. These lipid changes were found only in potato species that were able to acclimate to cold. Recent studies by Palmgren et al. (1988) suggest that increases in plasma membrane ATPase activity can be brought about by increases in 18:2 fatty acid. These results, combined with our findings listed above, suggest that membrane lipid changes may be playing an important role in modulation of membrane ATPase during cellular response to low-temperature stress.

A scheme outlining the events leading to stress-induced injury or 


\section{STRESS}

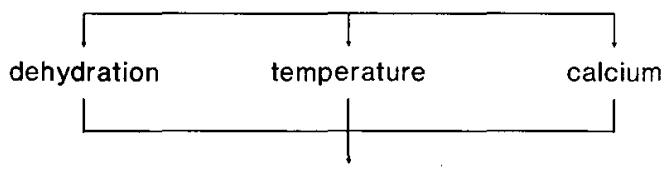

MEMBRANE LIPIDS

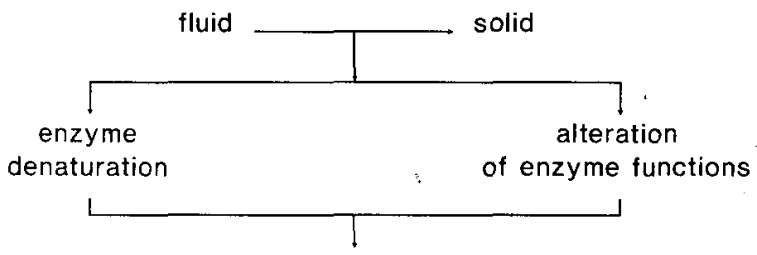

\section{CELLULAR RESPONSE}

Fig. 5. A flow diagram illustrating membrane lipids as a site of membrane/ cellular response to stress. Changes in the physical state of membrane lipids from liquid crystalline (fluid) to solid gel can result in cascade of reactions leading to the cellular response.

\section{INJURY OR RECOVERY}

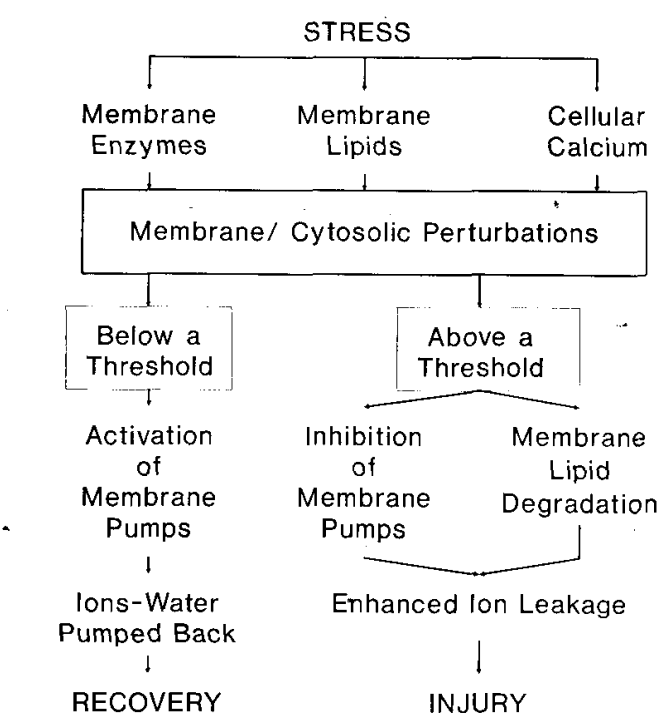

Fig. 6. Sequence of events leading to injury or recovery following stressinduced perturbations in membrane enzymes/membrane lipids/cellular calcium.

recovery is given in Fig. 6. Stress-induced changes in the membrane enzymes, in membrane lipids, and/or in cellular calcium can lead to membrane/cytosolic perturbations. If these perturbations are below a threshold, recovery occurs via activation of membrane pumps that transport leaked ions and solutes back into the cell. Water follows by osmosis back into the cell, leading to the disappearance of the soaked condition (recovery). If these perturbations are above a threshold, the activity of the pumps is reduced/lost and further deterioration of membrane lipids also occurs. These changes result in enhanced leakage (injury) and eventual death of the cell (Fig. 6).

Role of light in environmental stress. Injury to the photosynthetic apparatus can occur whenever light energy trapped within the chloroplasts exceeds the capacity of the plant to process (thus dissipate) this light energy (Powles, 1984). The injury is brought about by photoinhibition and photo-oxidation. Under environmental stress conditions, an impairment of the enzymatic capacity to use trapped energy can create these damaging conditions (Osmond, 1981). In a recent study, we have found that a cold-sensitive potato species (Solanum tuberosum L.) suffers much greater photoinhibition and impairment of photosynthetic apparatus at low temperature and moderate light level than a cold-tolerant species (S. commersonii Dun.) (Steffen and Palta, 1986). We also found that tolerant species had two layers of palisade cells in their leaves, compared to only one layer in the sensitive species (Palta and Li, 1979). It appears that the tolerant potato species are able to protect themselves from photoinhibition, in part, by filtering out the light in the multiple palisade layers and thereby reducing the effective amount of light reaching the leaf interior. In another study, we investigated the effect of light stress following various levels of freezing stress on photosynthetic capacity (Steffen and Palta, 1989). Light treatment during the post-thaw period resulted in inhibition of the photosynthetic process compared to dark treatment. These results underscore the importance of considering light as a stress component in the study and understanding of stress interactions.

\section{Future directions of research}

Further research at the cellular and membrane level is needed to understand stress interactions. While several scientists are actively pursuing research on the plant response to individual stresses, very little is being done to investigate stress interactions. From the current knowledge of plant response to environmental stresses, it appears that the following areas of research might lead to a better understanding of interactions among various stresses: a) Study the role of calcium in mediating stress response. Calcium appears to be linked to stress-induced perturbations, including biological stress. The discovery of calmodulin and the possibility that calcium can act as a second messenger has opened new opportunities to investigate stress interactions. b) Investigate biochemical and biophysical changes during recovery from stress. While the literature is full of research on the aspects of injury and acclimation to stress, little has been done to investigate the mechanisms of recovery following stress. From our work, it is clear that plants have the capability to recover from stress-induced injury. By understanding changes during recovery, we might gain knowledge of the initial perturbations by (the locus of) injury. c) Study perturbations of cellular membranes, especially changes in lipid-protein interactions during stress. There is considerable evidence supporting membranes as key sites of stress response. Most studies to date have focused either on membrane lipids or membrane proteins. Recent research suggests that changes in lipid-protein interactions maybe important in mediating stress responses. d) Investigate and characterize the common proteins that are produced during acclimation to several environmental stresses. Recently, several research groups have identified new proteins that are produced during acclimation to heat (heat shock proteins), cold, water, and salinity stress. One good way to understand stress interactions will be to find out if some common proteins are produced when plants are subjected to different stresses. The challenge in this area of research will be to understand functions of these new proteins, This information will be valuable in understanding the genetic basis of stress interactions. e) Study the mechanisms of light interactions with other environmental conditions. Even moderate light levels can become excessive (photo-oxidative) under stressful conditions. Little attention has been paid to understand how plants have developed mechanisms to dissipate excess light energy under stressful conditions. Knowledge of how light may play a role in injury, recovery, and acclimation will be valuable in understanding stress interactions under natural conditions.

\section{SUMMARY AND CONCLUSIONS}

A common feature among several environmental stresses is dehydration. The process of dehydration and the stresses that result from it are similar for water stress and extracellular freezing stress. However, for osmotic stress, dehydration and the accompanying stresses are very different. Lack of understanding of the basic similarities and differences among these three stresses has misled researchers in the recent past.

One approach to understanding the relationship among stresses is to study the initiation and progression of injury at the cellular and membrane levels. The common visual symptoms of injury among several stresses, i.e., water-soaked appearance and/or enhanced 
leakage of solutes, suggest injury to the cell membranes. The nature of membrane perturbations following freezing stress has been investigated recently. It has been demonstrated that freeze-thaw stress results in alteration of membrane transport properties. This alteration is an early manifestation of the injury. It has also been shown that injury is gradual and reversible. These and other results support the hypothesis that early events in freezing injury involve functional and/or structural changes in the membrane associated $\mathrm{H}^{+}$-ATPase (membrane pump). In addition to direct impact of stress on enzymes, the cellular response to stress can be brought about by changes in the membrane and cytosolic calcium concentration and by changes in the fluidity and composition of membrane lipids. All of these possibilities can be combined to explain a sequence of events leading to either repair/recovery or lethal injury to the cell.

The following areas of investigation might help explain/understand interactions among various environmental stresses: a) study the role of calcium in mediating stress response during injury, recovery from injury, and acclimation to stress; b) study the perturbations of membranes, especially membrane lipid-protein interactions, by stress; c) study functions of common proteins produced during acclimation to several types of environmental stresses; d) study how irradiance interacts with other environmental stresses under natural conditions during injury, recovery, and acclimation.

\section{Literature Cited}

Arora, R. and J.P. Palta. 1986. Protoplasmic swelling as a symptom of freezing injury in onion bulb cells.. Its simulation in extracellular $\mathrm{KCl}$ and prevention by calcium. Plant Physiol. 82:625-629.

Arora, R. and J.P. Palta. 1988. In vivo perturbation of membrane-associated calcium by freeze-thaw stress in onion bulb cells. Simulation of this perturbation in extracellular $\mathrm{KCl}$ and alleviation by calcium. Plant Physiol. 87:622-628.

Ashworth, E.N., G.A. Davis, and J.A. Anderson. 1985. Factors affecting ice nucleation in plant tissues. Plant Physiol. 79:1033-1307.

Carruthers, A. and D.L. Melchior. 1986. How lipid bilayers affect membrane protein activity. Trends Biochem. Sci. 11:331-335.

Cramer, G.R., A. Lauchli, and V.S. Polito. 1985. Displacement of $\mathrm{Ca}^{2+}$ by $\mathrm{Na}^{+}$from the plasmalemma of root cells. Plant Physiol. 79:207-211.

Dowgert, M.F. and P.L. Steponkus. 1984. Behavior of the plasma membrane of isolated protoplasts during a freeze-thaw cycle. Plant Physiol. 75:1139.

Hepler, P.K. and R.O. Wayne. 1985. Calcium and plant development. Annu. Rev. Plant Physiol. 36:397-439.

Iswari, S. and J.P. Palta. 1989. Plasma membrane ATPase as a site of functional alteration during cold acclimation and freezing injury, p. 123137. In: P.H. Li (ed.). Low temperature stress physiology in crops. CRC Press, Boca Raton, Fla.

Kuiper, P.J.C. 1985. Environmental changes and lipid metabolism of higher plants. Physiol. Plant. 64:118-122.

Legge, R.L., J.E. Thompson, J.E. Baker, and M. Lieberman. 1982. The effect of calcium on the fluidity and phase properties of microsomal membranes isolated from postclimacteric golden delicious apples. Plant\& Cell Physiol. 23:161-169.

Levitt, J. 1980. Responses of plants to environmental stresses. Chilling, freezing, and high temperature stresses. vol. 1. Academic, New York.

Lynch, J., G.R. Cramer, and A. Lauchli. 1987. Salinity reduces membraneassociated calcium in corn root protoplasts. Plant Physiol. 83:390-394.

Lyons, J.M. and C.M. Asmundson. 1965. Solidification of unsaturatedsaturated fatty acid mixtures and its relationship to chilling sensitivity in plants. J. Amer. Oil Chem. Soc. 42:1056-1058.

Lyons, J.M. and J.K. Raison. 1970. Oxidative activity of mitochondria isolated from plant tissues sensitive and resistant to chilling injury. Plant Physiol. 45:386-389.

Lyons, J.M., T.A. Wheaton, and H.K. Pratt. 1964. Relationship between the physical nature of mitochondrial membranes and chilling sensitivity in plants. Plant Physiol. 39:262-268.

Marine, D. 1982. The role of $\mathrm{Ca}^{2+}$ and calmodulin in plants. What's new. Plant Physiol. 13:37-40.

Marme, D. 1983. Calcium transport and function, p. 599-625. In: A. Lauchli and R.L. Bieleski (eds.). Encyclopedia of plant physiology. vol. 15. Springer-Verlag, Berlin.

Osmond, C.B. 1981. Photorespiration and photoinhibition: Some implica- tions for the energetic of photosynthesis. Biochim. Biophys. Acta 639:7798.

Paliyath, G., B.W. Poovaiah, G.R. Munske, and J.A. Magnuson. 1984 Membrane fluidity in senescing apples: Effects of temperature and calcium. Plant \& Cell Physiol. 25:1083-1087.

Palmgren, M.G., M. Sommarin, P. Ulvskov, and P.L. Jorgensen. 1988. Modulation of plasma membrane $\mathrm{H}^{+}$-ATPase from oat roots by lysophosphatidylcholine, free fatty acids and phospholipase $\mathrm{A}_{2}$. Physiol. Plant. 74:11-19.

Palta, J.P., K.G. Jensen, and P.H. Li. 1982. Cell membrane alterations following a slow freeze-thaw cycle: Ion leakage, injury and recovery, $\mathrm{p}$. 221-242. In: P.H. Li and A. Sakai (eds.). Plant cold hardiness and freezing stress. Mechanisms and crop implications. Academic, New York.

Palta, J.P. and O.Y. Lee-Stadelmann. 1983. Vacuolated plant cells as ideal osmometer: Reversibility and limits of plasmolysis, and estimation of protoplasm volume in control and water-stress-tolerant cells. Plant Cell Environ. 6:601-610.

Palta, J.P., J. Levitt, and E.J. Stadelmann. 1977a. Freezing injury in onion bulb cells: I. Evaluation of the conductivity method and analysis of ion and sugar efflux from injured cells. Plant Physiol. 60:398.

Palta, J.P., J. Levitt, and E.J. Stadelmann. 1977b. Freezing injury in onion bulb cells: A comparison of freezing vs. dessication and living vs. dead cells. Physiol. Plant. 41:273-279.

Palta, J.P. and P.H. Li. 1978a. Cell membrane properties in relation to freezing injury, p. 93-115. In: P.H. Li and A. Sakai (eds.). Plant cold hardiness and freezing stress. Mechanisms and crop implications. Academic, New York.

Palta, J.P. and P.H. Li. 1978b. Examination of ultrastructural freeze-injury in the leaf cells of tender and hardy potato species. HortScience 13:387.

Palta, J.P. and P.H. Li. 1979. Frost hardiness in relation to leaf anatomy and natural distribution of several Solanum species. Crop Sci. 19:665670.

Palta, J.P. and P.H. Li. 1980. Alterations in membrane transport properties by freezing injury in herbaceous plants. Evidence against rupture theory. Physiol. Plant. 50:169-175.

Palta, J.P. and L.S. Meade. 1987. Fatty acid composition changes after cold acclimation: Comparison of leaf, callus, and purified membranes. Plant Physiol. 83:71. (Suppl.)

Pearce, R.S. 1988. Extracellular ice and cell shape in frost-stressed cereal leaves: A low temperature scanning-electron-microscopy study. Planta 175:313-324.

Poovaiah, B.W. 1985. Role of calcium and calmodulin in plant growth and development. HortScience 20:347-352.

Poovaiah, B.W. and A.S.N. Reddy. 1987. Calcium messenger systems in plants. CRC Crit. Rev. Plant Sci. 6:47-102.

Powles, S.B. 1984. Photoinhibition of photosynthesis induced by visible light. Annu. Rev. Plant Physiol. 35:15-44.

Schaller, G.E. and M.R. Sussman. 1988. Phosphorylation of the plasma membrane $\mathrm{H}^{+}$-ATPase of oat roots by a calcium-stimulated protein kinase. Planta 173:509-518.

Singer, S.L. and G.L. Nicholson. 1972. The fluid mosaic model of the structure of ceil membranes. Science 175:720-731.

Steffen, K.L., R. Arora, and J.P. Palta. 1989. Sensitivity of photosynthesis and respiration to a freeze-thaw stress: Role of realistic freeze-thaw protocol. Plant Physiol. 89:1372-1379.

Steffen, K.L. and J.P. Palta. 1986. Effect of light on photosynthetic capacity during cold acclimation in a cold-sensitive and a cold-tolerant potato species. Physiol. Plant. 66:353-359.

Steffen, K.L. and J.P. Palta. 1989. Light stress following a frost episode influences the frost tolerance of a wild potato species. J. Amer. Soc. Hort. Sci. 114:656-661.

Steponkus, P.L. 1984. Role of the plasma membrane in freezing injury and cold acclimation. Annu. Rev. Plant Physiol. 35:543.

Steponkus, P.L. and S.C. Weist. 1978. Plasma membrane alterations following cold acclimation and freezing, p. 75. In: P.H. Li and A. Sakai (eds.). Plant cold hardiness and freezing stress: Mechanisms and crop implications. Academic, New York.

Steponkus, P.L., R.Y. Evans, and J. Singh. 1982. Cryomicroscopy of rye mesophyll cells. Cryoletters 3:101.

Sze, H. 1985. H-translocating ATPases: Advances using membrane vesicles. Annu. Rev. Plant Physiol. 36:175.

Tao, D., P.H. Li, and J.V. Carter. 1983. Role of the cell wall in freezing tolerance of cultured potato cells and their protoplasts. Physiol. Plant. 58:527-532. 